by the voluntary bodies which fought the Bill at Committee stage. It seems odd that there is no mention of this and some other important milestones, including the establishment of the NCC/ Ministry of Defence Advisory Committee on Foulness and of the Thames Estuary Wildlife Conservation Group. The authors do give credit, however, to the conservation efforts of the PLA, the Central Electricity Generating
Board, and others. By encouraging them, their book can only help conservation which depends so much on the foresight and responsibility of all involved in land-use decisions.

John Andrews is Head of the Conservation Planning Department of the Royal Society for the Protection of Birds, dealing with land-use issues affecting birds throughout the UK.

\section{Baffling disease}

\section{H. Andrewes}

Influenza: The Viruses and the Disease. By Sir Charles H. Stuart-Harris and G. C. Schild. Pp. $x+242$. (Arnold: London, 1976.) £12.

THE remarkable properties of the causative virus have made influenza a most fascinating yet baffling disease. Despite a vast amount of work there is still much to learn, particularly about how the infection can be controlled. Stuart-Harris and Schild's book is packed with information. For much of his working life, Sir Charles StuartHarris has been involved with the disease; he has in particular cultivated the area in which clinical and laboratory medicine overlap. Dr Schild was until recently in charge of the WHO World Influenza Centre at the National Institute for Medical Research, London, UK. Each of 12 chapters is followed by a list of references varying in number from 40 to over 100. Even these, as the preface points out, represent only a selection from the vast world literature.

We have first an outline of "the modern history of the study of the causes of human influenza". The authors pay only brief attention to work preceding the discovery, in 1933 , of the susceptibility of ferrets to infection. Indeed there is only passing mention of Pfeiffer's so-called influenza bacillus, Haemophilus influenzae. Nor is much space devoted to outbreaks in earlier years, for the identity of the infecting agent of those times can only be inferred. Even the catastrophic 1918-19 pandemic is not considered at length.

Having reviewed the most important laboratory findings, including transmission to animals, cultivation and interference, chapter 2 discusses in great detail the molecular biology and replication of the virus. There are many excellent and well reproduced electron micrographs. These depict the whole virus and its separated components, and are supplemented by diagrams clearly explaining the structure and mode of replication of the virus.
Description of the viral antigens leads on to discussion of virus variation, the minor changes known as 'antigenic drift' and the periodical major 'antigenic shift' that herald pandemics. We learn how two viruses growing together in culture or in living animals may exhibit recombination of characters, resulting in viruses with hybrid properties.

Chapter 4 deals with the influenza viruses of swine, horses, birds and other species; for it is now recognised that man is only one host of a versatile family of viruses. There is increasing interest in the possibility that new pandemic strains may arise from recombination between human and animal viruses. The authors are, however, very cautious and write: "At the present time is seems somewhat premature to assess the significance of these findings....".

Chapter 5 describes the disease in man and its complications. We then have two chapters on epidemiology, with numerous diagrams illustrating the variations in mortality and morbidity from year to year. The authors admit to being puzzled as to where the virus goes between outbreaks and why older viruses disappear when new antigenic variants appear. There are full references to the work that led to the doctrine of "original antigenic sin". This concerns the remarkable fact that a person's first experience of a virus determines the pattern of his subsequent antibody responses: these are largely directed against the virus he first encountered rather than against more recent ones. When tests are made in the light of this, against various animal viruses, all sorts of intriguing questions arise about the nature of the influenza viruses involved in outbreaks of earlier years.

Studies of immunity, next described, deal largely with the relation between immunity and the level of antibodies in serum and in nasal secretions. Clearly it is vital to understand these matters if vaccination is to be attempted with any success. The results of several trials with inactivated vaccines of various kinds are reviewed in detail, and there is discussion about the best strategy for vaccine administration.

There are obvious theoretical advan- tages in the use of a live attenuated vaccine: it can be given as a spray and thus be much more acceptable; and it would be vastly cheaper. Methods used for virus attenuation are described together with the results of trials; those on a large scale have been carried out mainly in the USSR and Jugoslavia. It seems that, although acceptably attenuated vaccines are available, the results of trials have been variable. The authors suggest that live vaccines are more likely to be effective "when a new pandemic virus is triggering off a new cycle of epidemics". Otherwise, killed vaccines may be better.

"Some progress" is reported in the field of chemoprophylaxis and chemotherapy. Amantidine, in particular, is discussed, as is the theoretical basis of the ways in which drugs may contrive to upset replication or release of virus.

The final chapter provides details of the various techniques in use for the isolation and assay of virus, and of the various serological methods in use. Particularly useful will be the accounts of the recently described "single-radialimmunodiffusion" and "single-radialhaemolysis" tests.

A postscript discusses the recent excitement over the small outbreak due to a swine-like virus in New Jersey. As is wisely pointed out, "spread of swine influenza viruses from pig to man and their restricted spread in the population may have occurred from time to time in the past without detection"

The book will be of great value to workers in this field and to those concerned with preventive measures. It invites comparison with another, much smaller, popular book on the subject, Influenza, the last great plague by Professor W. I. B. Beveridge (Heinemann Educational: London, £2.95). Stuart-Harris and Schild's book is full of well documented facts, but the authors are very chary of even modest speculation. Not so, Professor Beveridge; he freely discusses pandemics from 1700 onwards, considers the birth of pandemics, and seems to have little doubt about the role of animal viruses in their genesis. Whereas one book describes the "trees" in most valuable detail, the other makes a bold attempt to discern the nature of the "wood".

The only serious criticism of StuartHarris and Schild's book is that the print is annoyingly smaller than usual; doubtless the price would otherwise be even higher. One hopes that the rising cost of books will not cause publishers to lower their standards of presentation.

Sir Christopher Andrewes was Deputy Director of the National Institute for Medical Research, London, UK, from 1952-61, during which time he was also in charge of the WHO World Influenza Centre. 